\title{
Design and prototype of mobile robots for rescue operations E. Faruk Kececi*
}

\author{
Department of Mechanical Engineering, Izmir Institute of Technology, Urla, Izmir 35430, Turkey
}

(Received in Final Form: August 21, 2008. First published online: September 30, 2008)

\begin{abstract}
SUMMARY
This study concerns the design and prototype of four different mobile robot platforms for rescue robot operations after an earthquake. At first, a test field is constructed to represent a mildly damaged earthquake zone. The test field consists of eight different sections: sand, gravel, ditch, water, bridge, incline, decline, and turn. The mechanical structure, electronics, software, communication, and possible sensory systems are explained. After the robots are manufactured, they are physically tested for their performance in the test field for 18 different parameters. The test results show the effective body structure. Challenges of the rescue robot design are explained and future expectations are given.
\end{abstract}

KEYWORDS: All terrain; Prototype robot design; Rescue robotics.

\section{Introduction}

Rescue robotics focuses on designing robots to help people after disasters. When time and equipments are limited, robots can be used to save lives, and emergency responders do not need to risk their own lives during the rescue operations. One of the most common disasters for which rescue robots are being used is earthquake. The general requirements and expectations for rescue robots are given in refs. [1-8].

In mobile robot applications, the mission time, wireless operating distance, rough terrain capability, and fall durability become important limitations of the design. For rescue robots, since the robot needs to penetrate into the rubble, additional limitations to mobile robot are required; the rescue robot should be small and light enough not to disturb unstable objects in the rubble.

The early efforts of rescue robotics started with the RoboCup Rescue competition to increase interest and research in the field. ${ }^{9,10}$ The main problem of rescue robot design is to have a mobile base which can go over on a rough terrain. The concept of rough terrain is described in refs. [9-11], where the damage to the building is described with different color of courses from yellow to orange to red, illustrating the terrain difficulty from light to moderate to complex.

When rescue robots are categorized, the locomotion of the robots is mostly either as tracked vehicles ${ }^{12,13}$ or snaketype robots. ${ }^{14,15}$ It is also suggested that if they can change their shapes, this will assist them to climb and maneuver in confined spaces. ${ }^{16}$ The reason a wheeled robot cannot be

\footnotetext{
* Corresponding author. E-mail: farukkececi@iyte.edu.tr
}

used easily in rescue operations is that, the robot will have less ability to overcome the obstacles because of the fact that a wheel cannot go over an obstacle bigger than its radius.

However, in order to combine the advantages of wheeled and legged drive systems, leg-wheel structures are created. ${ }^{17,18}$ In this type of design, a wheel is attached at the end of the leg structure. When the robot is on a flat surface, it can use the wheel to go faster. When the wheels cannot be used because of the obstacles or surface irregularities (such as sand) the wheels are locked and the legs are used to pass the region. The difficulty of such a design is that the wheel at the end of the leg causes too much weight and requires the leg motors to be larger.

The mechanical design advantage of a snake-type robot is that it can be small in cross section, which enables the robot to penetrate into the rubble easily compared to a tracked vehicle. ${ }^{14,15,19}$ However, the snake-type robots are more difficult to control and their load to body weight ratio is smaller than a tracked-type robot. Most of the time, this type of a robot is used for observation. Especially some of the snake robot designs ${ }^{20,21}$ required to use small wheels under the each segment of the robot. Unfortunately, this type of a mechanical structure does not work inside rubble since the dirt fills into the gaps too easily.

Special designs, such as a ball structure, ${ }^{22}$ are also tried. This type of a robot could penetrate into rubble; however, since the robot cannot move actively they cannot cover all the rubble and a system needs to be developed to track the position of the robot inside the ruble. A similar idea is achieved by a core-bored robot, but this types of robot can only provide visual feedback to the areas it can reach. ${ }^{23}$

There are also more advanced designs where each side of the robot has a track and this design allows the robot to move no matter which of its surfaces is in contact with the ground. ${ }^{24}$ Micro robotics has also been used in debris; however, this type of a system requires many robots to be effective. ${ }^{25}$ Small humanoid robots can be used in victim exploration, but the servo motor technology needs to be improved for this type of robot to be strong enough to maneuver on rough terrain. ${ }^{26}$ Adding one degree of freedom arm on the robot also helps to balance the robot when it is working in the rubble. ${ }^{27}$

At the World Trade Center, existing mobile robots were used for surveillance, but most of these robots were designed for military applications, not specifically for rescue operations in an earthquake zone. ${ }^{12,28,29}$

In earlier studies, the problems encountered with mobile robots in a rescue field are explained, but the design process of the robot is not mentioned. This paper explains the mechanical design procedure and prototype stages of four 
different types of mobile robots which are designed for a mildly damaged earthquake zone. Moreover, the robots are evaluated for their performance and the design challenges in the procedure of robot design are summarized.

\section{Problem Definition}

The biggest problem in rescue robot design is that the rubble after an earthquake is not standard, and for any given rubble it can be completely different. However, the damage is described as light, mild, and heavy depending on the broken things and fallen appliances. ${ }^{11}$

Apart from the geometrical difficulties, the objects in any house become obstacles for the robots. Even though some of these obstacles such as chairs, books, or even sofas can be guessed, places like kitchens or offices are harder to model. In any kitchen, the glassware and china breaks and forms odd shape obstacles. In the offices, little office supplies, such as a paper clip or papers create very difficult obstacles to pass.

In this study, the problem is described in two parts: the field and the limitations on the robot. The described field represents a mildly damaged building after an earthquake where small things have fallen on the floor but not the big appliances. The limitations on the robot force the robot to be on a reasonable scale to the obstacles. If there are no physical limitations on the robot, the natural intention will be making the robot as big as possible to overcome any obstacle. However, in general, robots for rescue operations should be small to penetrate the rubble better and should be light in order not to apply too much pressure on trapped people, or unstable parts of the building.

\subsection{The test field}

The test field to represent the earthquake zone is designed to have different types of terrain properties. The field is shown in Fig. 1 and the details of the terrain are explained later. Wooden boxes of dimensions $50 \mathrm{~cm}$ to $100 \mathrm{~cm}$ are made and these boxes are filled with sand, gravel, and water. Between the gravel-filled box and water-filled box, a space is used as a ditch where the falling and climbing abilities of the robots are tested.

Sections of the test field are as follows:

1. Sand terrain: Traction control of the designed robot on a soft terrain is the purpose of this section. The sand grain size is smaller than $1 \mathrm{~mm}$.

2. Gravel terrain: Gravel size is smaller than $30 \mathrm{~mm}$, which acts like broken objects after an earthquake.

3. Falling into the ditch: The robot needs to fall $20 \mathrm{~cm}$ and during this fall it should not get damaged mechanically or electronically.

4. Climbing up the ditch: The robot needs to have the ability to climb over a 20 -cm straight wall.

5. Water pool: In order to test the water resistance ability of the robot, the robot needs to pass the water pool which has a 5-cm depth.

6. Passing under the bridge: In order to limit the total height, the robot should travel under the bridge with a height of $12 \mathrm{~cm}$.

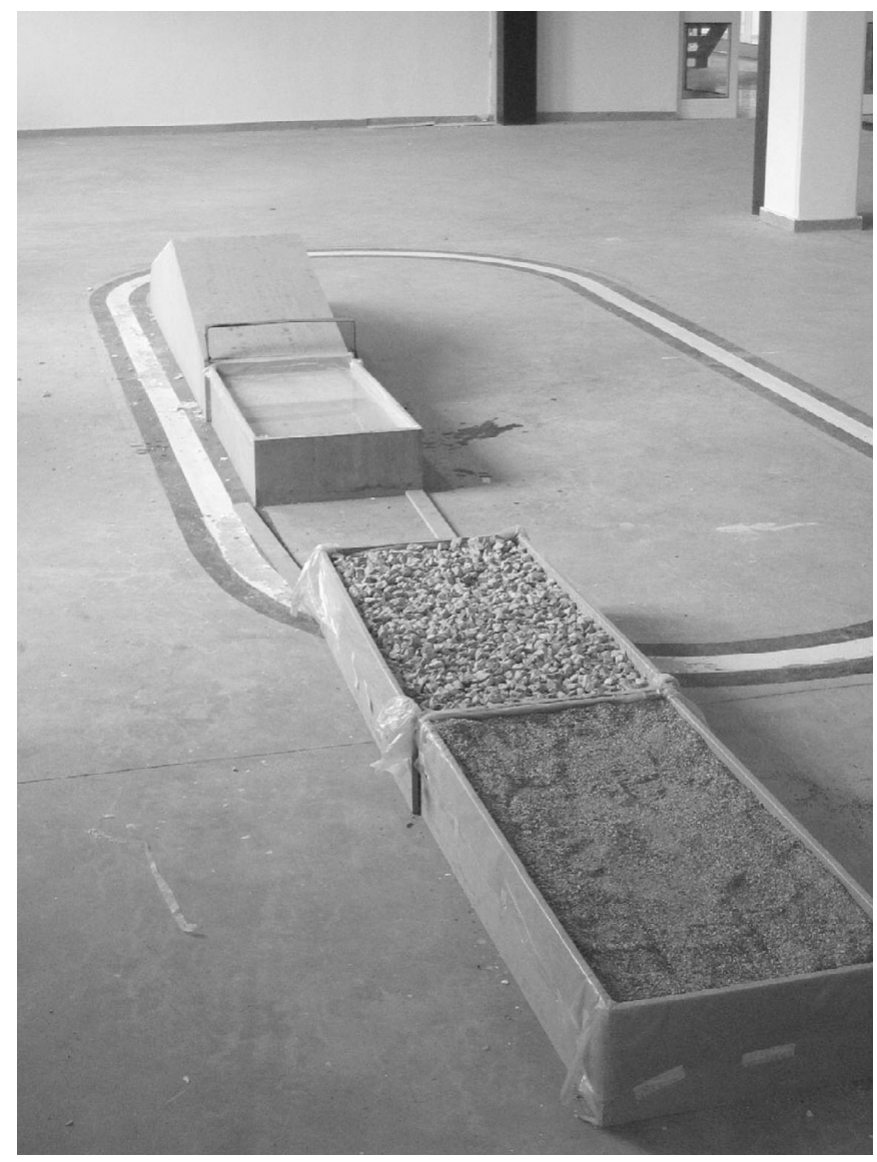

Fig. 1. The track and its different sections.

7. Inclined surface: The robot should climb a $15^{\circ}$ ramp, which requires extra engine power.

8. Peak: The ground clearance of the robot becomes important at this stage for the robot not to get stuck at the peak point.

9. Declined surface: During the travel on the declined surface the robot should have breaking ability not to fall.

10. Turn: The steering ability of the robot is tested, where the robot needs to make a $U$ turn with a radius of $1.5 \mathrm{~m}$.

\subsection{Robot limitations}

The limitations on the robot were:

1. Size: The robot must be smaller than A4 size paper $(210 \mathrm{~mm} \times 297 \mathrm{~mm})$, but there is no initial height limitation.

2. Load: The robot needs to carry a load (120 $\mathrm{mm} \times$ $50 \mathrm{~mm} \times 20 \mathrm{~mm}$, length $\times$ width $\times$ depth; and $120 \mathrm{~g})$.

3. Trajectory: The robot should go through each field.

4. Control: The robot needs to be remote controlled.

When the limitations on the robot are considered with the field, there are some limitations which are not explicitly mentioned. Different terrain types on the test field require the robot to have a durable locomotion system. Falling down and climbing $20-\mathrm{cm}$ height requires a suspension system and a climbing mechanism. In order to be able to turn, the robot either should have a steering mechanism or it should skid 

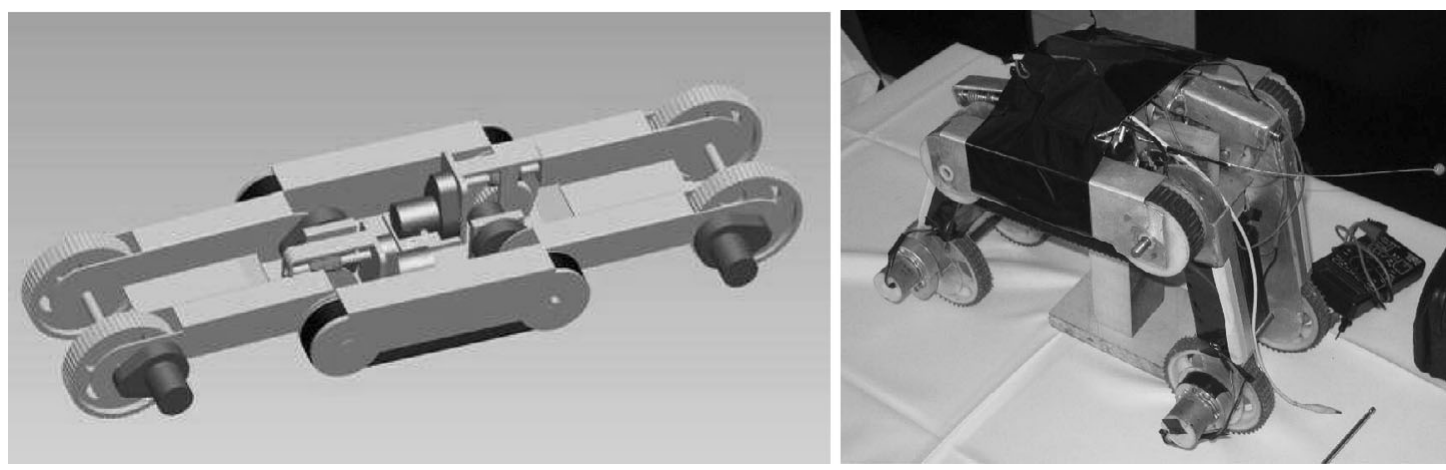

Fig. 2. Design of the tracked robot with three segments and prototype robot.
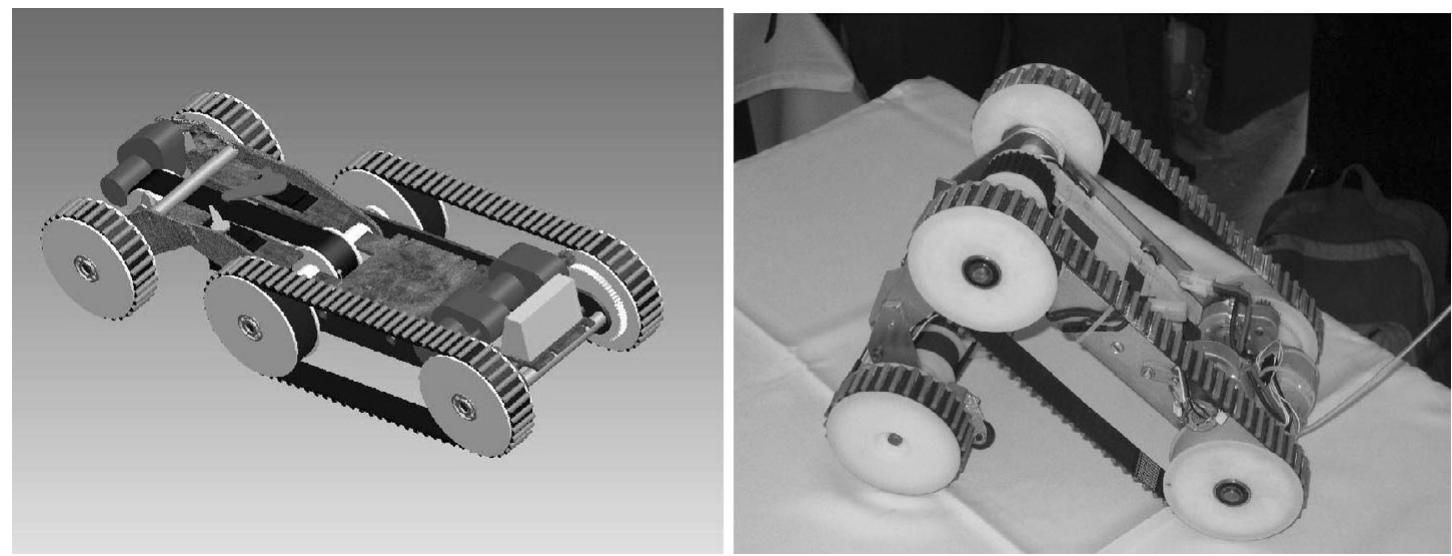

Fig. 3. Design of the tracked robot with lifting tail and prototype robot.
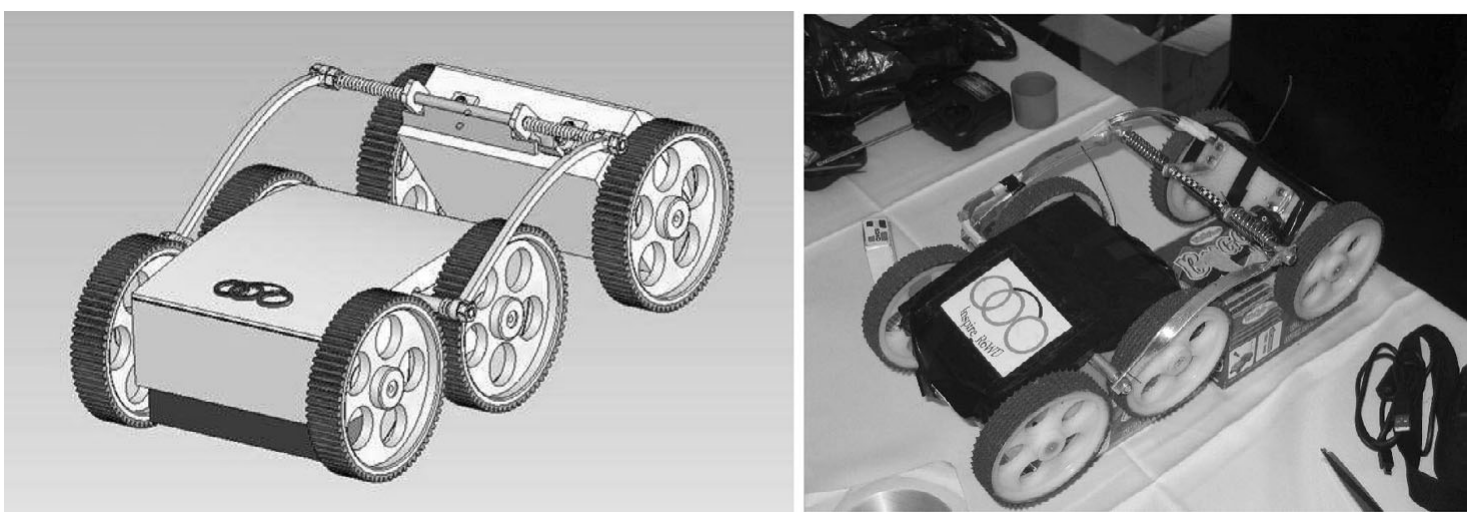

Fig. 4. Design of the rocker robot and prototype robot.

steer. The size of the robot compared to the distance it should climb and the bridge height it should pass under limits use of big wheels or tracks. Carrying the load imposes the center of the gravity to be calculated very carefully.

\section{Prototypes}

When the prototypes are being designed and built, the robot limitations are kept in mind and they are designed to pass the test field. The following subsections explain the mechanical, hardware, software, sensory, and control systems of the robotic platforms.

\subsection{Mechanical design}

In the mechanical design stage, six different solutions are found. One of these solutions included a hook to throw to the bridge and pull the robot out of the ditch. Another design used a rocket engine to fly down to and out of the ditch. These ideas are rejected, because the control of the system is thought to be too difficult.

The other four solutions are preceding and the design pictures and actual robots are showed in Figs. 2-5.

The three-segment tracked vehicle shown in Fig. 2, designed to have driving motors for the left side tracks placed toward the front side, and for the right side placed in the back. This design ensures that the weight of the motors is 


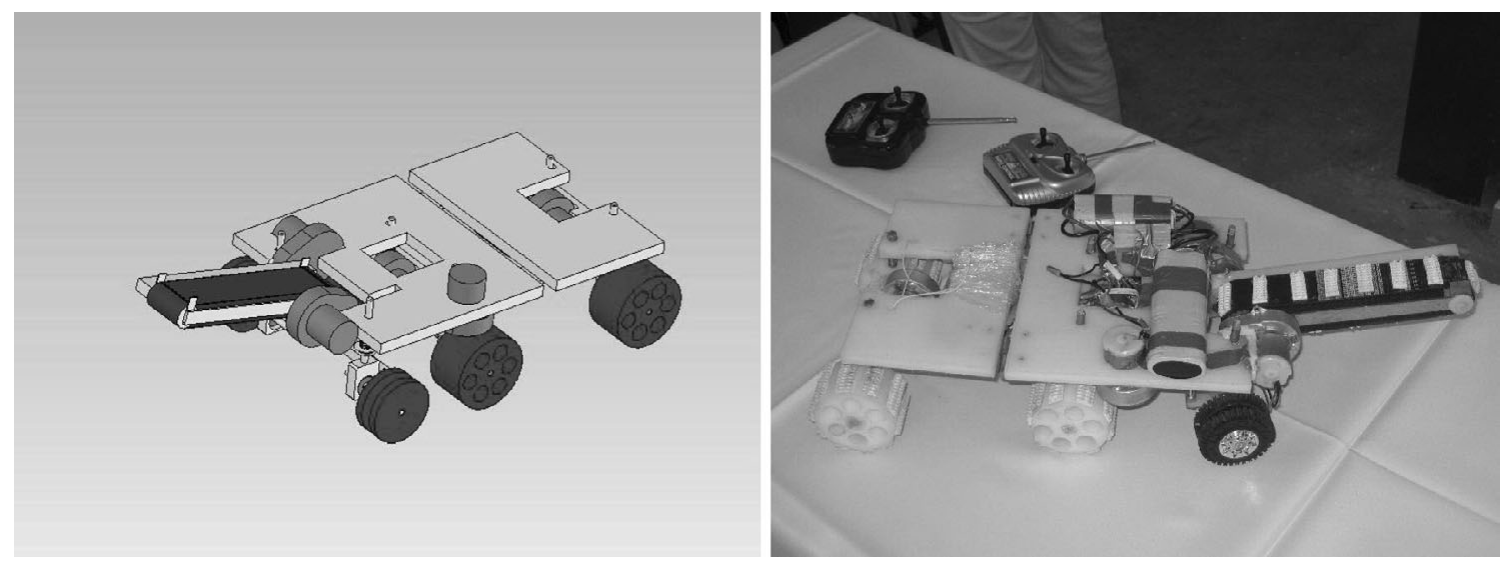

Fig. 5. Design of the wheeled robot with lifting horn and prototype robot.

distributed equally. Electronic equipment is placed in the middle because the middle can be lifted while the robot is passing through the water pool. Batteries are positioned at the first and third segments because of the lack of space on the middle segment but the connections of the batteries are kept close to the middle segment so that the batteries can be merged into water up to their connection level.

The main body of the three-segment tracked robot could not be manufactured with milling operation, because the maximum depth of the cutting tool was not enough. This restriction forced the main body part to be manufactured by folding operations. After the aluminum plate was cut with a laser cutter, it was folded with a folding machine.

On the tracked vehicle with lifting tail design (Fig. 4), in order to protect the electronics, the electronic circuits are placed inside a protective layer, namely inside a balloon. In this design, two servo motors connected with a linkage mechanism are used to lift the tail, since using two motors instead of one distributes the weight and space of the actuators evenly on the tail part. Since it was not possible to find a track with teeth at both sides for the design in Fig. 5, a timing belt was improved by gluing rubber pieces to have teeth on the outer side of the belt.

The rocker robot has $6 \times 6$ drive ability and it can go upside down. However, the six motors and the battery on the robot take three-fourth of the space inside robot; therefore, there is an insufficient amount of space for the robot to carry a functional load.

The wheeled robot with lifting horn has six wheels, an Ackerman steering system and a leverage system for lifting the robot's body. The body flexibility is considered to create constant contact between the wheels of the robot and the ground during the climbing of the ditch. However, it was this flexibility which caused the robot skid and slide when it was in motion.

Initially these four robots were tested to see if they could pass the minimum requirements of an earthquake rescue robot. These requirements are: mobility and accurate positioning and orientation. The testing was based on each robot completing an elliptical path. This test proved that wheeled robots were not able to meet the minimum requirements. Both robots had problems with skidding and sliding; therefore, their design is considered unremarkable and further testing was not performed.

\subsection{Hardware and communication system software}

The hardware of the tracked robots consists mainly of five parts: actuators, remote control, motor drivers, microcomputer, and battery.

Figure 6 shows the electronic circuit for the tracked robot with three segments. Where six $120 \mathrm{rpm} 12 \mathrm{~V}$ DC motors are controlled. Two of these motors are used to lift the end segments whereas the other two motors are used for rotating the wheels at the end of these sections clockwise and counterclockwise. The main body of the robot is driven with tracks by two motors. The microcontroller, PIC16F877, controls six motors through six LMD18200T 3 A, 55V $\mathrm{H}$ bridges. Seven thousand eight hundred and five voltage regulator (KA7805) provides the $5 \mathrm{~V} \mathrm{DC}$ to the circuit. The commands from the user are transferred to the robot with a remote controller, which is connected to the PORTB3-6 pins of the microcontroller.

The tracked robot with lifting tail has two standard servo motors and three $120 \mathrm{rpm} 12 \mathrm{~V} \mathrm{DC}$ motors. The DC motors are used to drive the left and right tracks as well as the back wheels. The tracks are used both in driving backward and forward as well as turning. The rear wheels only assist in backward and forward driving. The servo motors are connected to each other and lift a small idle wheel at the back to lift the rear wheels during the turns, ensuring that the robot turns about its main body's geometrical center. This turning point allows the robot to have less friction during the turns.

Figure 7 shows the circuit diagram for the tracked robot with lifting tail. A PIC16F877 microcontroller drives three LMD18200T $\mathrm{H}$ bridges and two servo motors. The two servos are driven simultaneously, so they are connected to the same pin of the microcontroller. Seven thousand eight hundred and five voltage regulator provides $5 \mathrm{~V}$ DC to the circuit.

In order to control the robots, a five-channel 33-MHz FM remote controller is used. This remote controller consists of two parts: the transmitter and the receiver. The operator uses the transmitter to send the desired commands to the robot and the receiver placed on the robot catches the signals 


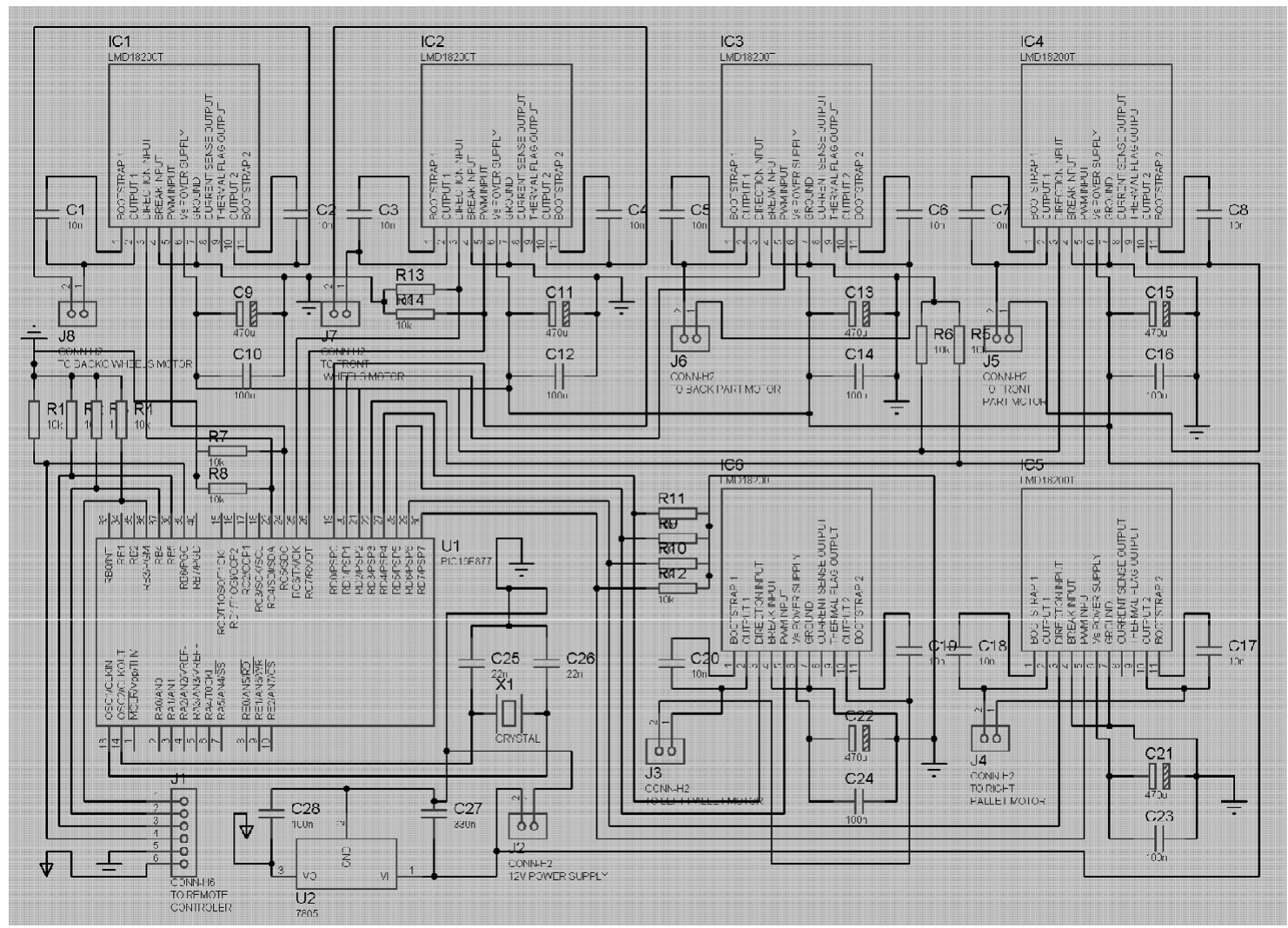

Fig. 6. Electronic circuit of the tracked robot with three segments.

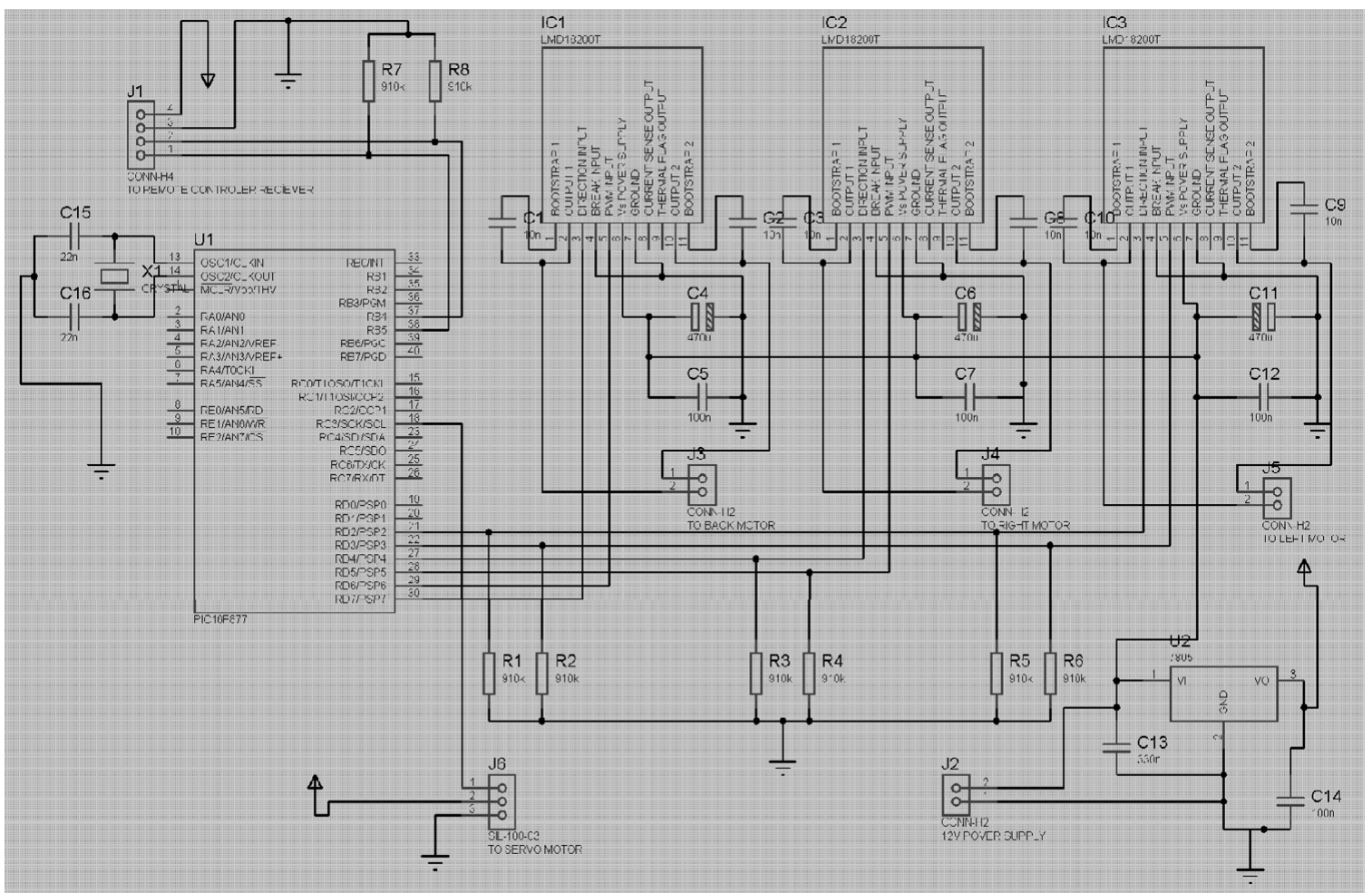

Fig. 7. Electronic circuit of the tracked robot with lifting tail.

and sends them to the microprocessor to be decoded. The microcontroller controls the different motors depending on the received signals.

On the three-segment robot, four channels of the remote controller are used and this allows the robot to go forward and backward, turn left and right, rotate the front and back wheels, and lift the first and third segment of the robot together or separately.

On the tailed robot, only two channels of the controller are used. The first channel commands the robot to turn right or 
left, whereas the second channel is used to send the signals to make the robot go forward or backward.

In both of the robot circuits, the receiver voltage, namely $5 \mathrm{~V} \mathrm{DC}$, is supplied from the robots' batteries.

On the three-segment robot circuit, there are six LMD18200 $\mathrm{H}$ bridges 3A 55V drivers. These $\mathrm{H}$ bridges allow the motor to be driven in the desired direction. Since the motors are actuated in $12 \mathrm{~V} \mathrm{DC}$, the $\mathrm{H}$ bridges are supplied with $12 \mathrm{~V} \mathrm{DC}$. The direction pin of a driver is connected to the microcontroller, and by sending logic 1 or 0 the motor rotates clockwise or counterclockwise.

Since the tailed robot has two servo motors, the microcontroller is used to drive these motors as well as three $12 \mathrm{~V}$ DC motors. The three $12 \mathrm{~V}$ DC motors are driven by LMD18200 H bridges. Servo motor signals come directly from the microcontroller. Since these motors are mechanically connected to each other, they are also electrically connected to each other. DC supply voltage of $5 \mathrm{~V}$ is provided from a 7805 voltage regulator.

A PIC16F877 microprocessor is used as the micro controller which runs at $4 \mathrm{MHz}$. The microcontroller decodes the signals coming from the receiver and controls the motors.

For different positions of the different channels, different tasks of robot movements are assigned to the microcontroller, and the incoming signal is decoded and the motors are actuated depending on the operator's request.

Rechargeable battery of $12 \mathrm{~V} 7 \mathrm{Ah}$ is used as a main power supply and another $12 \mathrm{~V} 1 \mathrm{Ah}$ battery is used for powering the electronics.

\subsection{Sensors}

The sensors can be placed on a rescue robot and should be categorized in two sections: internal and external.

3.3.1. Internal sensors. Encoders, accelerometers, gyroscopes, magnetic compasses, tilt, and shock sensors can be used as internal sensors. An inertial navigation system can be built by using these sensors to understand the position and the orientation of the robot.

The main problems will be skipping and sliding and compensating for these errors will provide more accurate location of the robot.

\subsubsection{External sensors. The following sensors can be used} as external sensors: gas, smoke, oxygen, light, temperature, humidity, ammonia, ultrasound, microphone, camera, laser distance, and listening system.

The ultrasound sensors control the size of the tunnel the robot is moving in so that the robot can avoid getting stuck. A listening system can be employed to locate trapped people. A camera can be used to receive visual feedback, whereas a microphone can be used to listen to the debris. A laser distance sensor located next to the camera can give information on depth; however, the frequency of this sensor should be such that it should not harm the eyes of the victims and rescue personnel. An ammonia sensor can be used to locate victims, and the other sensors; gas, smoke, oxygen, light, temperature, and humidity give information about the environment.

With a speaker located on the robot, the operator can talk to the victims and learn more about their situation, other possible victims as well as the debris. Lighting should be employed on the robot to ensure that the camera provides clear images.

Although external sensors are not included in the design process of the current robots, in later versions of these rescue robots the size, weight, placement, battery requirements, operating temperature, and conditions of the external sensors should be taken into account.

Two batteries should be placed on the robot to protect the robot from the power surges. When the robot is forced to pass an obstacle, the motors pull too much current, causing electric cuts for the electronics. In order to ensure continuous power for the electronic circuit and the sensors, these elements should be supplied from a different battery. The main battery supplies power to the motors.

\subsection{Communication systems}

In this research, FM wireless communication is used for the ease of the remote controller, namely a model plane controller. In this type of controller, the number of channels determines the maximum controllable motors at a given time. However, the distance becomes a problem; if it is less than $100 \mathrm{~m}$, the signal gets distorted because of the noise, especially coming from the antennas.

An RF wireless system can be used if the distance requirement is higher. The wireless systems require decoding at the receiver side.

When the cable connection is considered, the data transfer can be serial or parallel. During serial communication, 1 bit is used as a start bit and 1 bit used as an end bit and in between 1 byte of data is transferred over one cable. If there will be a feedback from the robot, there should be another cable for feedback signals. Parallel communication requires different cables for each bit to be transferred. For the feedback signals there should be other sets of wires. This type of communication is faster, since in one step of time the data can be transferred; however, it requires $n$ number of cables for $2^{n}$ data, whereas serial communication uses only one cable for sending data and one for receiving data.

\subsection{Strategy of control}

Simple feed forward control is used in this study, where the operator sees the environment from a camera located on the robot and sends commands to the motors on the robot.

This control algorithm is improved by using imageprocessing techniques to understand the image better for clues in the rubble. ${ }^{30}$

The robot can also include reflexive actions to find its path. ${ }^{31}$ This type of control can also be used for a robot to protect itself, when the operator commands the robot to move and if that type of motion will cause the robot to get stuck, the control system can measure the opening of the tunnel and can stop the command to execute in order to protect the robot.

Eventually, the most desirable control strategy would be complete autonomy, where the robot finds the victims itself. Considering the randomness of a debris, autonomy inside rubble seems difficult to achieve, but not impossible. 


\section{Testing Procedure}

In order to test the effectiveness of the robot designs, the test field shown in Fig. 1 is built and the robots are tested for their performance. During the testing 18 different design parameters for a rescue robot are considered and they are as follows:

1. Overcoming geometrical difficulties: On a difficult terrain, defined in Section 2, it is desired that the robot be able to go over as many obstacles as possible.

2. Volume capacity: If there is more space inside the robot, it can be used for carrying different sensors.

3. Weight capacity: If the weight carrying capacity is larger, the robot can transport more necessary equipment inside the earthquake zone.

4. Energy necessity: Lower energy requirement decreases the size of the power source which will result in a lighter and smaller robot.

5. Reverse fall: The ability to move upside down will allow the robot to accomplish its task after flipping.

6. Failure durability: If the robot consists of less parts and simple mechanisms, it will have fewer tendencies to fail.

7. Weight: The weight of the robot itself is desired to be less.

8. Dimensions: Dimensions of the robot body are desired to be small.

9. Clearance: Height of the robot from the ground should be as much as possible.

10. Maneuver capability: The driving ability of the robot increases the robot's ability to travel in confined spaces.

11. Problem with other systems: The mechanical or electronic systems of the robot should not interfere with each other.

12. Falling resistance: From what height the robot can fall and not have any mechanical or electrical problem determines the falling resistance.

13. Velocity: Speed of the robot should be high.

14. Number and size of motors: The number and size of the motors used on the robot are responsible for determining the battery requirement of the system.

15. Body flexibility: A robot with a flexible body will be able to go through confined spaces easily.

16. Control difficulty: Depending on the driving method, the number of motors, body flexibility, and the degrees of freedom needing to be controlled should be less in order to ensure ease of control.

17. Programming ease: The software of the robot should be simply written so that it can be easily updated.

18. Manufacturing ease: The physical manufacturing of the robot should be simple to allow cheap mass production of the robot.

Depending on the importance of the parameters, a percentage is assigned for each parameter. For this design study, the parameter values are shown in Table I. The user parameters and robot abilities received higher percentage while the design and manufacturing parameters received lower percentage, since hard work of the designer, ease of use of the robot, and higher abilities of the robot are expected outcomes of the project.
Table I. Percentage values of the design parameters.

\begin{tabular}{llc}
\hline Design parameters & $\begin{array}{c}\text { Importance } \\
\text { percentage }(\%)\end{array}$ \\
\hline 1 Overcoming geometrical difficulties & 12 \\
2 Volume capacity & 8 \\
3 Weight capacity & 8 \\
4 Energy necessity & 8 \\
5 Reverse fall & 8 \\
6 Failure durability & 8 \\
7 Weight & 8 \\
8 Dimensions & 5 \\
9 Clearance & 5 \\
10 Maneuver capability & 5 \\
11 Problem with other systems & 5 \\
12 Falling resistance & 5 \\
13 Velocity & 5 \\
14 Number and size of motors & 2 \\
15 Body flexibility & 2 \\
16 Control difficulty & 2 \\
17 Programming ease & 2 \\
18 Manufacturing ease & 2 \\
\hline
\end{tabular}

\section{Experimental Results}

The tracked robot with three segments has both the tracks and the wheels as driving elements. On sand and gravel, the tracks are used to receive higher traction forces. When the first and third section is lifted, the robot can go like a wheeled robot and achieve higher speeds. The other use of the first and third segments is that when the robot is falling into the ditch and climbing out, these parts are used to elevate the robot body. Having six motors makes the robot very capable of maneuvering in tight spaces; however, the energy requirement of the robot is very high and the synchronous control of the robot requires an experienced driver. The robot weighs $4.7 \mathrm{~kg}$ and it can carry up to $3 \mathrm{~kg}$. After it expands, its dimensions are $660 \mathrm{~mm} \times 251 \mathrm{~mm} \times 71 \mathrm{~mm}(l \times w \times h)$. On wheels, it can achieve a speed of $19 \mathrm{~m} / \mathrm{min}$; with its tracks, it can go $13 \mathrm{~m} / \mathrm{min}$. By using skid steering it can turn about its own geometrical center.

The tracked robot with lifting tail uses tracks and wheels to move forward. In order to use skid steering to maneuver, the back part, in other words the tail, is lifted with servo motors and the linkage mechanism. The tail is necessary, when the robot is climbing out of the ditch, to keep the contact and provide traction force. The belt under the tail part helps the robot to climb into the water section of the track by providing force at the upper edge. The robot weighs $4.4 \mathrm{~kg}$ and it can carry up to $7 \mathrm{~kg}$. After it expands, its dimensions are $510 \mathrm{~mm} \times 195 \mathrm{~mm} \times 115 \mathrm{~mm}(l \times w \times h)$. In full speed, on a flat surface, it can achieve a speed of $13 \mathrm{~m} / \mathrm{min}$. The minimum steering radius, the shortest radius the robot can turn around itself, is $65 \mathrm{~cm}$ from its center of gravity to the center of the circle.

For the tracked robot with lifting tail it took $1 \min 9 \mathrm{~s}$ to complete the track, whereas the three-segment robot completed it in $1 \mathrm{~min} 32 \mathrm{~s}$. These running times are calculated by averaging 10 trials. The reason that the three-segment robot completed the track at a lower speed was because it takes too much time for the segments to move. Otherwise, 
Table II. Robot performances.

\begin{tabular}{llcc}
\hline Properties & $\begin{array}{c}\text { Tracked robot with } \\
\text { three segments }\end{array}$ & $\begin{array}{c}\text { Tracked robot with } \\
\text { lifting tail }\end{array}$ \\
\hline 1 Weight $(\mathrm{kg})$ & 4.7 & 4.3 \\
2 & Dimensions $(l \mathrm{~mm} \times$ & $660 \times 251 \times 71$ & $510 \times 195 \times 115$ \\
$\quad$ & $w \mathrm{~mm} \times h \mathrm{~mm})$ & & 7 \\
3 & Load capacity $(\mathrm{kg})$ & 1.5 & 34 \\
4 & Clearance $(\mathrm{mm})$ & $23-228$ & 13 \\
5 & Speed $(\mathrm{m} / \mathrm{min})$ & $13-19$ & 65 \\
6 & Steering $(\mathrm{mm})$ & 0 & 23 \\
7 & Incline angle $(\mathrm{deg})$ & 35 & \\
\hline
\end{tabular}

when the robot is using the wheels it is 1.5 times faster than the lifting tail robot.

The test results and the robot performances are summarized in Table II. Table III shows the evaluation of the robots with respect to the design parameters.

Since the three-segment robot can lift the center part, it has a great advantage on clearance. However, the lifting ability limits the load carrying capacity drastically. By moving the segment independently, this robot can shift its center of gravity and can achieve inclines at higher angles.

\section{Discussions}

During the experiments, different problems occurred and these problems taught the design team how to design better robots. These problems are detailed later and they include: placement of motors, gearboxes and power transmission elements, battery selection, mechanical jamming, and obstacle navigation.

At first, the tracked robot with three segments had a failure when it fell into the ditch. The motor actuating the front wheel hit the sidewall and the gearbox was damaged and this experience led to improving the robot by placing the motors toward the inner body of the robot.

The tracked robots are slower than the wheeled robots; however, they can carry heavier loads. An expected difficulty was that when the field gets wet the robots have a difficult time climbing the ditch. In later designs of rescue robots, wet floors should also be considered as obstacles. Another bottleneck in robot design is the gearboxes and power transmission elements. It was difficult to find small size gearboxes and power transmission elements, since in many robotic applications the size of the robot is not so crucial.

Next, weight shifting helps robots to climb greater angles as well as to slow down when it is coming to a ditch. If the robot does not sufficiently decrease its speed before a sharp decline, the impact will cause damages to the gearboxes.

The selection of battery also becomes very important, because when the robot gets stuck it starts to draw too much current and the battery cannot meet its need. This causes motors to stop. Especially the electronics should be fed from a different battery for power surge protection, because if there is no protection the electronics get reset.

The mechanical jamming occurred in the tracks, when sand and stone came in between the tracks and the driving pulleys. It was surprising to see that even grass caught in the tracks could stop the whole robot. In order to eliminate this failure the tracks should have idle wheels

Another unexpected problem was the performance drop of the robots caused by obstacles of office supplies. The robots performed very poorly when they were passing over paper piles. This failure occurred because of the obstacle not being stable. The same result is observed with pen and pencils and other small but unstable objects. The robot can pass the obstacle but skids and rotates unintentionally. This problem should be solved by including an inertial navigational system

Table III. Evaluation of the robot designs with respect to design parameters.

\begin{tabular}{|c|c|c|c|c|}
\hline & Design parameters & $\begin{array}{c}\text { Importance } \\
\text { percentage }(\%)\end{array}$ & $\begin{array}{l}\text { Tracked robot with } \\
\text { three segments }\end{array}$ & $\begin{array}{c}\text { Tracked robot with } \\
\text { lifting tail }\end{array}$ \\
\hline 1 & $\begin{array}{l}\text { Overcoming geometrical } \\
\text { difficulties }\end{array}$ & 12 & 12 & 12 \\
\hline 2 & Volume capacity & 8 & 8 & 6 \\
\hline 3 & Weight capacity & 8 & 4 & 5 \\
\hline 4 & Energy necessity & 8 & 2 & 6 \\
\hline 5 & Reverse fall & 8 & 8 & 0 \\
\hline 6 & Failure durability & 8 & 6 & 8 \\
\hline 1 & Weight & 8 & 2 & 6 \\
\hline 8 & Dimensions & 5 & 4 & 4 \\
\hline 9 & Clearance & 5 & 5 & 2 \\
\hline 10 & Maneuver capability & 5 & 4 & 3 \\
\hline 11 & Problem with other systems & 5 & 5 & 5 \\
\hline 12 & Falling resistance & 5 & 2 & 4 \\
\hline 13 & Velocity & 5 & 1 & 4 \\
\hline 14 & Number and size of motors & 2 & 1 & 2 \\
\hline 15 & Body flexibility & 2 & 2 & 1 \\
\hline 16 & Control difficulty & 2 & 0 & 2 \\
\hline 17 & Programming ease & 2 & 0 & 1 \\
\hline \multirow[t]{2}{*}{18} & Manufacturing ease & 2 & 1 & 2 \\
\hline & Total points & & 67 & 73 \\
\hline
\end{tabular}


inside the robot and measuring the skidding and sliding of the robot.

By changing the dimensions or combining different driving systems, these mobile robot platforms can also be used for other mobile robotic applications such as planetary explorations or any other terrain application. For instance, making these rescue robots, which are designed for an earthquake operation, fire proof will make them capable of working in a fire-fighting operation.

\section{Acknowledgments}

The author would like to thank the class of 2005 from the Department of Mechanical Engineering of Izmir Institute of Technology for their help and hard work in making these prototypes possible.

\section{References}

1. C. Schlenoff and E. Messina, "A Robot Ontology for Urban Search and Rescue," Workshop on Research in Knowledge, Bremen, Germany (2005) pp. 27-34.

2. L. Matthies, Y. Xiong, R. Hogg, D. Zhu, A. Rankin, B. Kennedy, M. Hebert, R. Maclachlan, C. Won, T. Frost, G. Sukhatme, M. McHenry and S. Goldberg, "A portable, autonomous, urban reconnaissance robot," Rob. Autonom. Syst. 40, 163-172 (2002).

3. F. Matsuno and S. Tadokoro, "Rescue Robots and Systems in Japan," IEEE International Conference on Robotics and Biomimetics, Shenyang, Liaoning, China (2004) pp. 12-20.

4. M. W. Kadous, R. K. Sheh and C. Sammut, "CASTER: A Robot for Urban Search and Rescue," Proceedings of the 2005 Australasian Conference on Robotics and Automation, Sydney, Australia (2005) pp. 1-10.

5. S. Tadokoro, T. Takamori, K. Osuka and S. Tsurutani, "Investigation Report of the Rescue Problem at Hanshin-Awaji Earthquake in Kobe," IEEE/RSJ International Conference on Intelligent Robots and Systems, Takamatsu, Japan (2000) pp. 1880-1885.

6. N. Ruangpayoongsak, H. Roth and J. Chudoba, "Mobile Robots for Search and Rescue," IEEE International Workshop on Safety, Security and Rescue Robotics, Kobe, Japan (2005) pp. 212-217.

7. S. Tadokoro, F. Matsuno, M. Onosato and H. Asama, "Japan National Special Project for Earthquake Disaster Mitigation in Urban Areas," Workshop on Synthetic Simulation and Robotics to Mitigate Earthquake Disaster, Padova, Italy (2003) pp. 22-27.

8. T. Takahashı and S. Tadokoro, "Working with Robots in Disasters," IEEE Robotics and Automation Magazine (2002) pp. 34-39.

9. H. A. Yanco and T. R. Balch, "The AAAI-2002 mobile robot competition and exhibition," AI Mag. 24(1), 45-50 (2003).

10. J. Casper and M. Micire, "The AAAI-2002 robot rescue," AI Mag. 24(1), 51-60 (2003).

11. K. Osuka, R. Murphy and A. C. Schultz, "USAR competitions for physically situated robots," IEEE Rob. Automat. Mag. 9(3), 26-33 (2002).

12. J. Carlson and R. R. Murphy, "How UGVs physically fail in the field," IEEE Trans. Rob. 21(3), 423-437 (2005).

13. R. R. Murphy, "Human-robot interaction in rescue robotics," IEEE Trans. Syst., Man Cybernet., Part C 34(2), 138-153 (2004).
14. J. Weston, "A safer way to search disaster sites", IEEE Rob. Automat. Mag. 7(3), 56-57 (2004).

15. I. Erkmen, A. M. Erkmen, F. Matsuno, R. Chatterjee and T. Kamegawa, "Snake robots to the rescue!," IEEE Rob. Automat. Mag. 9(3), 17-25, 2002.

16. R. R. Murphy, "Marsupial and shape-shifting robots for urban search and rescue," IEEE Intell. Syst. Their Appl. 15(2), 14-19 (2000).

17. M. Lacagnina, G. Muscato and R. Sinatra, "Kinematics, dynamics and control of a hybrid robot wheeleg," Rob. Autonom. Syst. 45, 161-180 (2003).

18. R. Siegwart, P. Lamon, T. Estier, M. Lauria and R. Piguet, "Innovative design for wheeled locomotion in rough terrain," Rob. Autonom. Syst. 40, 151-162 (2002).

19. Z. Yang, K. Ito, K. Hirotsune, K. Saijo, A. Gofuku and F. Matsuno, "A Mechanical Intelligence in Assisting the Navigation by a Force Feedback Steering Wheel for a Snake Rescue Robot," IEEE International Workshop on Robot and Human Interactive Communication, Kurashiki, Okayama, Japan (2004) pp. 113-118.

20. Y. Zhixiao, I. Kazuyuki, H. Kazuyuki, S. Kazuhiko and G. Akio, "A Mechanical Intelligence in Assisting the Navigation by a Force Feedback Steering Wheel for a Snake Rescue Robot," IEEE International Workshop on Robot and Human Interactive Communication, Kurashiki, Okayama, Japan (2004) pp. 113-118.

21. A. Crespi, A. Badertscher, A. Guignard and A. J. Ijspeert, "AmphiBot I: An amphibious snake-like robot," Rob. Autonom. Syst. 50, 163-175 (2005).

22. K. Kenji Inoue, M. Masato Yamamoto, Y. Yasushi Mae, T. Tomohito Takubo and T. Arai, "Design of Search Balls with Wide Field of View for Searching Inside of Rubble," IEEE International Workshop on Safety, Security and Rescue Robotics, Kobe, Japan (2005) pp. 170-175.

23. R. M. Voyles, A. C. Larson, J. Bae and M. Lapoint, "CoreBored Search-and-Rescue Applications for an Agile Limbed Robot," IEEE/RSJ International Conference on Intelligent Robots and Systems, Sendai, Japan (2004) pp. 58-63.

24. K. Tabata, A. Inaba and H. Amano, "Development of a Transformational Mobile Robot to Search Victims Under Debris and Rubble - 2nd Report: Improvement of Mechanism and Interface," IEEE International Workshop on Safety, Security and Rescue Robotics, Kobe, Japan (2005) pp. 19-24.

25. A. Himoto, H. Aoyama and O. Fuchiwaki, "Development of Micro Rescue Robot-Human Detection," IEEE International Conference on Mechatronics, Taipei, Taiwan (2005) pp. 526531.

26. T. Sugihara, K. Yamamoto and Y. Nakamura, "Hardware design of high performance miniature anthropomorphic robots," Rob. Autonom. Syst. 56, 82-94 (2008).

27. Y. Chiu, N. Shiroma, H. Igarashi, N. Sato, M. Inami and F. Matsuno, "FUMA: Environment information Gathering Wheeled Rescue Robot with One-DOF Arm," IEEE International Workshop on Safety, Security and Rescue Robotics, Kobe, Japan (2005) pp. 81-86.

28. A. Davids, "Urban search and rescue robots: from tragedy to technology," IEEE Intell. Syst. 17(2), 81-83 (2002).

29. R. R. Murphy, "Trial by Fire," IEEE Robotics and Automation Magazine (2004) pp. 50-61.

30. C. Castillo and C. Chang, "A Method to Detect Victims in Search and Rescue Operations Using Template Matching," IEEE International Workshop on Safety, Security and Rescue Robotics, Kobe, Japan (2005) pp. 201-206.

31. R. Chatterjee and F. Matsuno, "Use of single side reflex for autonomous navigation of mobile robots in unknown environments," Rob. Autonom. Syst. 35, 77-96 (2001). 
Reproduced with permission of the copyright owner. Further reproduction prohibited without permission. 